\title{
Digital "Cartes Synoptiques de la Chromosphere Solaire et Catalogues des Filaments et des Centres d'Activite"
}

\author{
Helen E. Coffey and Christine D. Hanchett ${ }^{1}$ \\ NOAA, National Geophysical Data Center (NGDC), World Data Center \\ A for Solar-Terrestrial Physics, 325 Broadway, Boulder, CO 80303 USA
}

\begin{abstract}
Almost 70 years ago Lucien d'Azambuja published the first "Cartes Synoptiques de la Chromosphere Solaire et Catalogue des Filaments de la Couche Superieure" (d'Azambuja 1928), a compendium of reduced solar observations covering the time period March 1919-January 1920. The compiled database gives both visual and quantitative measures of solar activity beginning with Carrington rotation 876 . Since then, data through 1989 have been published in succeeding Cartes Synoptiques issues. The World Data Center A (WDC-A) for Solar-Terrestrial Physics has digitized several long term solar publications, including the numerical text portion of the Cartes Synoptiques. We present an overview of this extraordinary historical solar database. WDC-A is using current technology to meet user requirements for data management, analysis and distribution, has compiled over 100 Megabytes of historical solar data and made it available over the Internet as part of a continuing data rescue effort. The data can be accessed via the World Wide Web at http://www.ngdc.noaa.gov/stp.
\end{abstract}

\section{Cartes Synoptiques}

Lucien d'Azambuja investigated solar filament and prominence behavior over many years, and maintained a synoptic program of this phenomena similar to the Zurich sunspot program. He made the connection that the filaments and prominences were the same phenomenon seen from different perspectives on the solar disk (d'Azambuja 1923). He contrasted the behavior of sunspot motions with filaments, noting similarities in rotation rate (equatorial filaments have speeds larger than the higher latitude filaments due to differential rotation of the sun) and lifetimes (d'Azambuja and d'Azambuja 1938). He noted that nearly one half of low latitude filaments suffer disparition brusques and disappear temporarily, reforming in nearly the same shape in $2 / 3$ of all cases (Tandberg-Hanssen 1995). His investigations also included solar-terrestrial effects, connecting the major solar flare of October 13, 1926 with the geomagnetic storm and aurora seen about 31 hours later (d'Azambuja and Grenat 1926), and papers on solar eclipses and comets.

\footnotetext{
${ }^{1}$ Under cooperative agreement with the Cooperative Institute for Research in the Environmental Sciences (CIRES), University of Colorado, Boulder, CO 80309 USA
} 


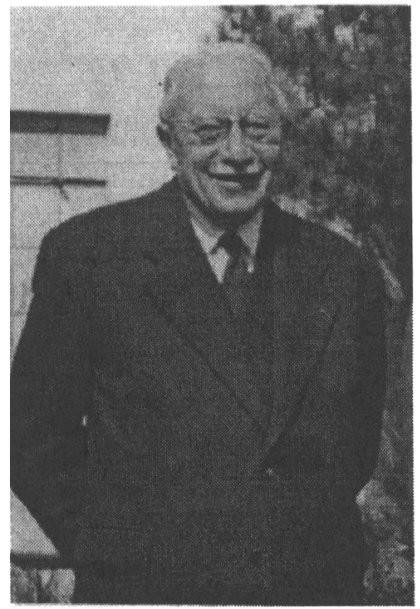

Figure 1. Lucien d'Azambuja, photographed in 1961 while working at McMath-Hulbert Observatory, University of Michigan. Courtesy of Helen Dodson Prince, reproduced with permission from Sky and Telescope, September 1970 issue.

D'Azambuja (Figure 1) left a legacy of an extensive historical solar filament database in the Cartes Synoptiques publications, which began with data for the year 1919 and continues to the present. In 1960 M. J. Martres assumed responsibility for the publication and maintained the continuing database. Early data include information about the number of days of observation of the filaments, their duration, their first and last appearance, the average coordinates, minimum and maximum length measurements, radial speed maxima, the importance ( 1 to 10 ), and general characteristics of the filaments. Several other kinds of statistics were included over the years. In 1945 d'Azambuja began a detailed listing of disparition brusques. With the International Geophysical Year (IGY) beginning in July 1957, a new format for the publication was introduced. The current catalog of filaments contains the filament number in order of decreasing longitude, coordinates of the center of gravity, importance ( 1 to 10 ), height of the prominence at the East and West limb, disparitions brusques observed on the disk, filaments with lifetimes greater than one rotation, and the number of transits and number of rotations where the filament has been identified. The current archive covers 70 years of solar observations, March 1919-December 1989, and totals 9.12 Megabytes. Figure 2 is a solar butterfly plot of the entire Cartes Synoptiques filament data archive. In total, there are 41,044 filament regions recorded. Each data point represents the Central Meridian Passage (CMP) date of a filament region crossing the solar disk. Note the "rush to the poles" close to solar maximum when the solar polar magnetic field reversal occurs.

Figure 3 shows the summary of importance values of filament regions for a given Carrington rotation for the 70 years covered by the the Cartes Synoptiques archive. Generally, one sees the lower importances at times of sunspot minima. 


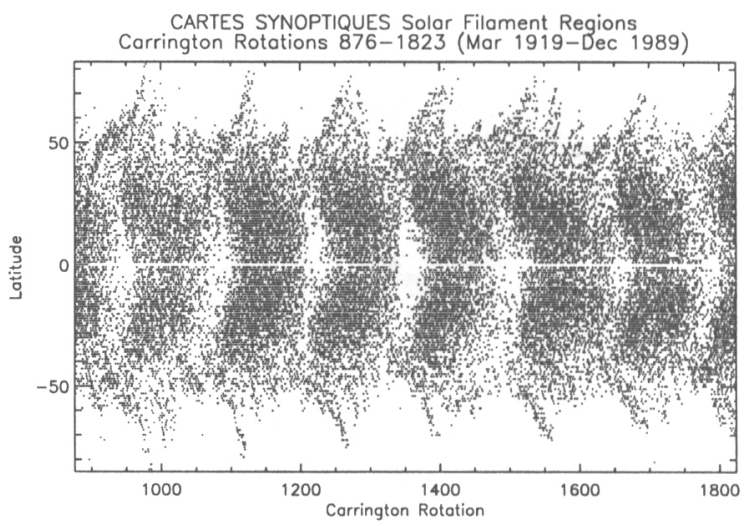

Figure 2. This 70 year solar butterfly plot of the Carte Synoptique solar filament regions archive by Carrington rotation shows the latitude distribution of CMP dates of filaments over seven solar cycles.

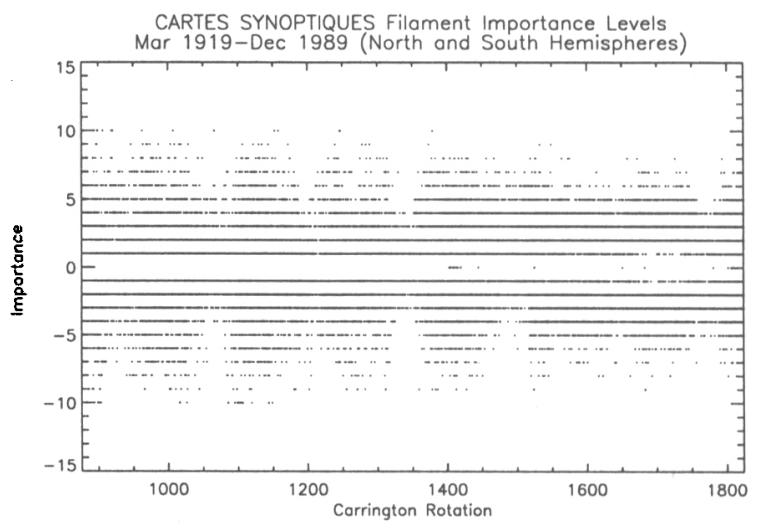

Figure 3. Importance values of filament regions versus Carrington rotation for the time period covered by the Cartes Synoptiques archive (solar cycles 15-22). Northern hemisphere filaments are assigned positive importance values, while southern hemisphere ones are assigned negative values. 
These importance values are especially low between cycles 18 and 19 (around 1954.3). In addition, there are asymmetries between the two hemispheres. Both hemispheres had low importance values during the 1954.3 minimum. However, the following cycle minimum in 1964.9 shows a predominance of the southern hemisphere contributing to the low values. Anomalously, during the solar cycle 21 maximum (1979.9), a lack of importance 1 values is seen in both hemispheres. Importances cluster around 2 to 5 with a few 6's and 7's seen. Several scientists have noted anomalous behavior during the 1980 time frame (Foukal 1997, private communication). Moreover, there is a general downward trend in large importance values over the 70 years with the most recent large cycles rarely above a value of 6 . Early cycles have several importance 10 values and substantial numbers of importance 7 and 8 . Perhaps the scaling procedure gradually changed over time.
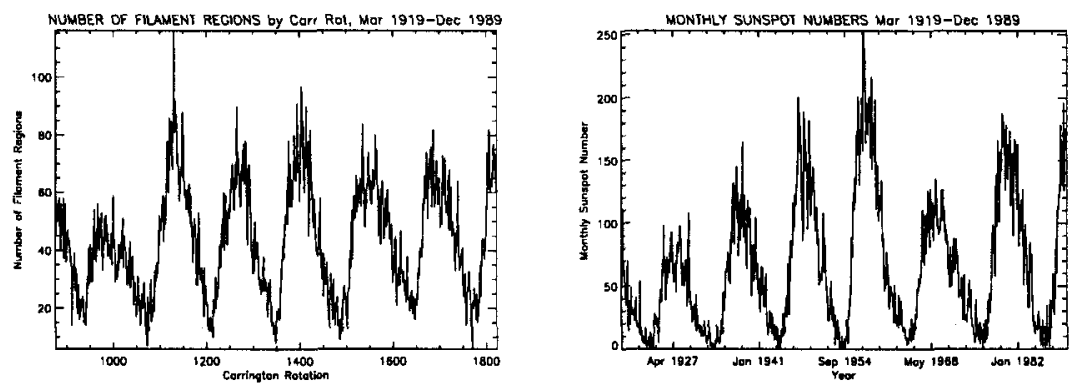

Figure 4. Left - the number of Cartes Synoptiques filament regions is plotted by Carrington rotation to show the distribution over 70 years. Right - for comparison we plot the Zurich monthly sunspot numbers.

In Figure 4 we show the number of filament regions from the Cartes Synoptiques archive and Zurich sunspot numbers (Sunspot Index Data Center) as a function of time. When these two plots are overlaid, one sees similar behavior, with some delay on the downward curve of the filament data after sunspot maximum, as noted by d'Azambuja and d'Azambuja (1948). However, if you shift the sunspot curve up about 18 units, the curves seem to fit together well. The number of filament regions appears to be more sensitive to solar minima than sunspot number. Perhaps one can use the filament minima behavior to predict the occurrence of sunspot minima and give advance information about the rate of rise of the new solar cycle.

Acknowledgments. This work was supported in part by the U.S.-Hungarian Joint Fund for Science and Technology, No. 95a-524. NGDC acknowledges with deep appreciation the efforts of many solar observers who contributed to the Cartes Synoptiques over many years. We expect to incorporate the solar digital databases into the Space Physics Interactive Data Resource (SPIDR) Data Base Management System. SPIDR allows the user to easily browse, plot, and download databases from http://www.ngdc.noaa.gov/stp.

This digital conversion work was funded in part by the NOAA Earth Systems Data Information Management (ESDIM) data rescue funds and by the NASA Space Physics Data System Data Set Preservation and Supply (SPDS) 
funds. NOAA contributed staff labor for this project.

\section{References}

d'Azambuja, L. 1923, Comptes Rendus, 176, 950

d'Azambuja, L. 1928, Annales de l'Observatoire de Paris, Section de Meudon, Tome VI, Fascicule I

d'Azambuja, L., and d'Azambuja, M. 1938, Comptes Rendus, 206, 819

d'Azambuja, L., and d'Azambuja, M. 1948, Annales de l'Observatorie de Paris, Section de Meudon, Tome VII, Fascicule VII

d'Azambuja, L., and Grenat, H. 1926, Comptes Rendus, 183, 701

Tandberg-Hanssen, E. 1995, The Nature of Solar Prominences, Kluwer Acad. Publ., Dordrecht, Holland 